\section{Cuidados paliativos para pacientes con infección por SARS-CoV-2/COVID-19; propuesta de un modelo de atención}

\author{
Palliative care for patients with SARS-CoV-2/COVID-19 \\ infection; proposal for a model of care
}

\author{
Dra. Diana P Pérez-Moreno,* Dr. Jorge Hernán López-Ramírez, \\ Dra. Catalina Torres-Espinosa ${ }^{\ddagger}$
}

RESUMEN. Los cuidados paliativos se convierten en la herramienta durante esta pandemia para brindar atención y apoyo a los pacientes, familiares y personal de la salud, controlando síntomas, promoviendo la calidad de vida, ayudando en la toma de decisiones difíciles y brindando atención integral de la salud física, psicológica, social y espiritual. Nuestro objetivo es presentar nuestro modelo institucional. Se realizó una revisión sistemática narrativa de la literatura disponible hasta el momento.

ABSTRACT. Palliative care becomes the tool during this pandemic to provide care and support to patients, family members and health personnel, controlling symptoms, promoting quality of life, helping in making difficult decisions and providing comprehensive health care, physical, psychological, social and spiritual. Our objective is to present our institutional model. A systematic narrative review of the literature available so far was performed.

\section{INTRODUCCIÓN}

A nte la pandemia por SARS-CoV-2/COVID-19 que enfrenta el mundo y nuestro país, los cuidados paliativos se convierten en una gran herramienta para brindar atención y apoyo a los pacientes, familiares y personal de la salud, controlando efectivamente síntomas, promoviendo la calidad de vida, ayudando en la toma de decisiones difíciles y brindando atención integral de la salud física, psicológica, social y espiritual. Este documento pretende compartir nuestro modelo institucional, entregando recomendaciones que apoyen a la toma de decisiones en pacientes con COVID-19 basadas en la literatura disponible a la fecha.

Una vez que ingresa el paciente a la institución se clasifica en menores de 65 años y mayores de 65 años, de acuerdo con el Servicio de Geriatría. Si el paciente es mayor de 65 años se valora el espacio vital por medio de preguntas de actividades básicas cotidianas, principalmente: 1 . ¿Hace un mes, el paciente era capaz de salir de casa solo y sin ayuda? Si la repuesta es "Sí» se clasifica como robusto. Si la respuesta es «No» se procede a indagar: ¿el paciente era capaz de movilizarse en casa solo sin ayuda? Si la respuesta es «Sí», clasificar como frágil e interrogar por antecedentes patológicos, marcadores de pronóstico vital como son: demencia, falla cardíaca, enfermedad coronaria, accidente cerebrovascular, enfermedad renal crónica estadío 4 o 5, neumopatía crónica que requiere de oxígeno, diabetes mellitus, etcétera. Si la respuesta es «No» clasificar como dependiente.

Los pacientes frágiles con uno o más factores de riesgo dados por las patologías previamente mencionadas o si el paciente es dependiente debe ser valorado y manejado por nuestro servicio.

\section{Revista Mexicana de \\ Anestesiología \\ Octubre-Diciembre 2020 \\ Vol. 43. No. 4. pp 288-295}

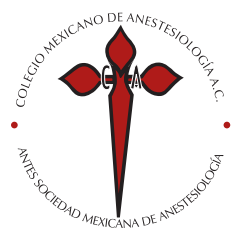

Palabras clave: Cuidados paliativos, medicina paliativa, pandemia, SARS-CoV-2/COVID-19.

Keywords: Palliative care, palliative medicine, pandemic, SARS-COV-2/COVID-19.

* Clínica de Dolor y

Cuidados Paliativos.

‡ Departamento de Geriatría.

Hospital Universitario Nacional de Colombia, Bogotá.

Solicitud de sobretiros: Dra. Diana P Pérez-Moreno Hospital Universitario Nacional. Calle 44 Núm. 69-75,

Bogotá, Colombia.

Teléfono: 4864234

Fax: 3904888

E-mail: d.perezmoreno@gmail.com

Recibido para publicación: 13-06-2020

Aceptado para publicación: 07-07-2020 
Una vez abordamos al paciente desde cuidados paliativos valoramos funcionalidad con:

\section{Escala ECOG (Eastern Cooperative Oncology Group)}

0: Completamente activo, capaz de realizar actividades previas a la enfermedad sin restricción.

1: Restringido en actividad física extrema, pero capaz de caminar y realizar trabajos livianos: oficina, trabajo en casa.

2: Capaz de caminar y autocuidado, pero incapaz de realizar cualquier trabajo. Deambula más de 50\% de las horas que está despierto.

3: Capaz de autocuidado limitado. Se mantiene limitado a cama o sillón más de $50 \%$ de las horas que está despierto.

4: Completamente incapacitado, no puede realizar autocuidado. Totalmente confinado a cama.

5: Muerte.

Y también valoramos el pronóstico vital con el índice pronóstico paliativo (PPI) $)^{(1)}$.

1. Índice de pronóstico paliativo (PPI: Palliative Prognostic Index)

El PPI no requiere de estudios de laboratorio, lo que hace más fácil su uso. La calificación total del PPI se calcula por la suma de cada una de estas variables y el rango es de 0 a 15 .

Cuando el PPI es mayor de 6 la supervivencia es menor a tres semanas y la sensibilidad es de $80 \%$, con especificidad del 85\%. Un PPI mayor a 4 la supervivencia es de seis semanas, la sensibilidad es de $80 \%$ y la especificidad del $77 \%$.

PPS (Palliative Performance Status): 10-20: 4.5, 30-50: 2, > 60: 0, Normal: 0 .

Síntomas clínicos: tolerancia a vía oral: moderada reducida: 1 , severamente reducida: 2.5 .

Presencia de edemas: 1.

Disnea en reposo: 3.5 .

Delirium: 4.5.

PPI: $>6$ supervivencia del paciente tres semanas.

PPI: $<4$ supervivencia seis semanas.

Se valorará si hace parte de alguno de los cinco grupos objetivos de cuidados paliativos de acuerdo con nuestro modelo referido más adelante.

Se aborda al paciente y a su familia por un grupo multidisciplinario conformado, entre otras profesiones, por: psicología, trabajo social, terapia ocupacional, psiquiatría, apoyo espiritual (Anexo 1).

Los pacientes con enfermedad grave que no se consideran candidatos a cuidados intensivos, es decir, aquéllos mayores de 65 años clasificados como dependientes o frágiles con marcadores de mal pronóstico, esto es, con múltiples co- morbilidades, enfermedades crónicas avanzadas e inclusive terminales oncológicas o no, tienen un riesgo muy alto de morir si adquieren la infección, con una tasa de mortalidad estimada de 15 a $22 \%{ }^{(2)}$, estos pacientes serán valorados y manejados por nuestro servicio.

Los pacientes oncológicos tienen mayor incidencia de eventos graves, es decir, muerte o ingreso en la UCI que requiere ventilación invasiva versus pacientes sin cáncer ( $8 \%$ vs 39\%; $p=0.0003)$ en el contexto de esta pandemia ${ }^{(3,4)}$.

\section{Los cinco grupos objetivo de nuestro modelo}

Pacientes con sospecha o diagnóstico de infección por COVID-19 y al menos una de las siguientes situaciones clínicas:

1. Población con enfermedades crónicas, progresivas, irreversibles e incurables que reciben manejo de soporte y adquieren la infección por COVID-19, ésta puede exacerbar la enfermedad coexistente, retar la reserva funcional y fisiológica lo suficiente como para producir el fallecimiento y/o requieren apoyo en manejo sintomático durante el curso de la enfermedad independiente de la severidad.

2. Población con o sin patologías crónicas de base que adquieren infección por COVID-19 en quien se requiere realizar triage y planificación del escenario de manejo, así como establecer conversaciones dificiles que ayuden a la coordinación de la atención, realizar voluntades anticipadas y establecer objetivos terapéuticos individuales.

3. Población con o sin patologías crónicas de base que adquieren SARS-CoV-2/COVID-19 y tiene voluntades anticipadas de disentimiento de medidas invasivas (ventilación mecánica, Unidad de Cuidados Intensivos, entre otros) que requiere manejo de soporte sintomático o con curso refractario al manejo médico y en quien se requiere reorientación de objetivos terapéuticos hacia medidas de confort.

4. Profesionales de la salud que atienden a paciente con infección por COVID-19 en los diferentes niveles de atención y requieren apoyo en la toma de decisiones dificiles, en la reorientación de los objetivos terapéuticos y en la comunicación de malas noticias.

5. Familiares de pacientes con infección por COVID-19 que requieren apoyo en conversaciones sobre la toma de decisiones, el intercambio de información clínica y pronóstica, así como el acompañamiento en la elaboración del duelo.

\section{Definiciones clave}

- Cuidados paliativos: la nueva definición propuesta por el consenso entre la Asociación Internacional de Hospicio y Cuidados Paliativos (IAHPC) y la Comisión Lancet es «Los cuidados paliativos son los cuidados holísticos activos 
de personas de todas las edades con graves sufrimientos relacionados con la salud debido a enfermedades graves y especialmente de aquellos cerca del final de la vida. Su objetivo es mejorar la calidad de vida de los pacientes, sus familias y sus cuidadores» ${ }^{(5)}$.

- Voluntades anticipadas: son disposiciones que una persona decide, estando en buenas condiciones de salud mental, sobre qué medidas y cuidados médicos quiere o no recibir en caso de tener una enfermedad grave.

Las familias y cuidadores del paciente deben participar en estas discusiones en la medida de lo posible y de acuerdo con los deseos de la persona. Esta es una práctica estándar en cuidados paliativos y de final de la vida.
En el contexto de infección por COVID-19/SARS-CoV-2, es probable que la persona presente un deterioro rápido, por lo que la oportunidad de debatir y participar en la toma de decisiones puede ser limitada o perdida.

El equipo de Cuidados Paliativos de la institución brinda este apoyo para la realización de este abordaje.

- Enfermedad crónica avanzada: enfermedad de curso progresivo, gradual, con diverso grado de afectación de la autonomía y de la calidad de vida, con respuesta variable al tratamiento específico, que evolucionará hacia la muerte a medio plazo.

- Síntomas refractarios: aquel que no puede ser adecuadamente controlado con los tratamientos disponibles, aplicados por médicos expertos, en un plazo de tiempo razonable.

Tabla 1: Estrategia del Servicio de Cuidados Paliativos de forma multidisciplinaria institucional durante la pandemia.

Capacidad convencional
Capacidad contingencia
Capacidad crisis

1) En la institución puede acceder al Servicio de Cuidados Paliativos especializados cinco días a la semana desde las 7 am hasta las $7 \mathrm{pm}$ por solicitud de interconsulta

2) En la institución puede acceder a clínica de dolor y cuidados paliativos especializados siete días a la semana desde $7 \mathrm{am}$ a $7 \mathrm{pm}$, por solicitud de interconsulta. Además, cuidados paliativos, disponibilidad telefónica las 24 horas del día, los siete días de la semana

3) Intervención de cuidados paliativos:

- Consultas para pacientes con mal pronóstico y en riesgo de intubación o reanimación priorizada

- Soporte para implementar voluntades anticipadas, ordenes de no reanimación, adecuación del esfuerzo terapéutico

- Integración de nuestros especialistas en dolor y cuidados paliativos con los diferentes servicios de la institución para ayudarlos a abordar a los pacientes con los siguientes criterios

- COVID-19 + con dificultad respiratoria, multimorbilidad, gravedad de la enfermedad, requerimiento de oxígeno

- Estado clínico: carga de síntomas, fragilidad uso clínico. Escala de fragilidad (geriatría), estado funcional basal ECOG, pronostico vital: PPI

- Estado de los códigos: voluntades anticipadas, orden de no reanimación, orden de no intubación, etcétera

Según la detección, sucederá lo siguiente:

- Reunirse con el paciente y con un familiar, representante legal o cuidador principal para realizar voluntades anticipadas y/o estado del código no intubación, no ventilación mecánica, no reanimación

- Entrenar al equipo de salud de la institución sobre voluntades anticipadas y documentos como no reanimación y no ventilación mecánica

- Ayudar con la documentación de las discusiones y el manejo de síntomas, incluidos los refractarios

- Evolución diaria para evaluar necesidades cambiantes para pacientes con COVID positivo, incluidos manejo de síntomas así como los refractarios, objetivos de atención, decisiones al final de la vida y abordaje a la familia en medidas de apoyo en elaboración de duelo

- Asesorar sobre el traslado a planes de atención domiciliaria, hospicios, hogares geriátricos

Después del horario de atención, el especialista de dolor cuidados paliativos puede ayudarlo por vía telefónica. Apoyo y coaching

PPI = Palliative Prognostic Index (Índice de pronóstico paliativo).

Fuente: los autores. 
¿Cuál es el enfoque o intervenciones que podemos brindar desde el Servicio de Cuidados Paliativos Institucional? (Tabla 1)

1. Manejo de síntomas, en especial los refractarios.

2. Voluntades anticipadas.

3. Abordaje de duelo y apoyo espiritual al paciente y a la familia.

4. Apoyo al personal de la salud.

Recomendaciones para el manejo de síntomas: en el contexto de la pandemia por COVID-19, para protección del personal de salud, recomendamos la administración de medicamentos en infusión continua utilizando el mejor dispositivo disponible: bomba de infusión PCA(Patient Controlled Analgesia/analgesia controlada por el paciente), bomba elastomérica, entre otros. El medicamento preparado tiene una duración de hasta 24 horas ${ }^{(6)}$. Los principales síntomas que van a requerir manejo de manera simultánea a los tratamientos de la enfermedad son en su orden: disnea, delirium, tos y fiebre.

Principios generales del uso de opioides que debemos recordar

1. La dosis correcta de opioides es la dosis que controla la disnea y el dolor, con un perfil aceptable de efectos secundarios (náuseas, vómitos, constipación, somnolencia). Recordar que la morfina no tiene dosis techo, pero se debe titular para definir la dosis óptima para el paciente.

2. La morfina es de manejo estándar, excepto en pacientes con insuficiencia renal crónica o lesión renal aguda, en los cuales debemos considerar otros opioides más seguros para el paciente renal.

3. Siempre comenzar con la dosis más baja posible en este escenario en particular en infusión continua ${ }^{(7)}$.

\section{Disnea}

Manejo de disnea moderada a severa en paciente con COVID-19: medidas no farmacológicas: la posición recta o inclinada hacia delante y hacer uso de técnicas de relajación. Usar máscaras reservorio, cánula nasal, pues son dispositivos que no aumentan el riesgo por producción de aerosoles ${ }^{(2)}$. Los opioides son la elección para el manejo de disnea en pacientes con síntomas moderados a severos, pero se deben considerar causas potencialmente tratables como infección bacteriana, derrame pleural, etcétera.

Esquema de manejo farmacológico: en casos severos, se puede realizar titulación rápida de morfina iniciando con $1 \mathrm{a}$ 3 mg cada 30 minutos vía SC o EV, hasta controlar la disnea, iniciar infusión $12 \mathrm{mg}$ en 24 horas ( $0.5 \mathrm{mg} / \mathrm{h}$ ) para casos leves y $24 \mathrm{mg}$ en 24 horas ( $1 \mathrm{mg} / \mathrm{h}$ ) en casos moderados. Usar rescates que sean iguales al $10 \%$ de la dosis total y titular de acuerdo con su necesidad. Vigilar la presencia de signos de efectos secundarios y en caso de presentarse, se debe reducir la dosis un $50 \%$ e hidratar ${ }^{(2)}$. Pacientes en fin de vida con síntomas refractarios considerar iniciar sedación paliativa (ver adelante).

Tos

Los mecanismos potenciales en pacientes con infección por COVID-19/SDRA incluyen inflamación, daño epitelial, impactación de moco y cambios neuromoduladores (aumento de la sensibilidad al reflejo de la tos). Como alternativas no farmacológicas se recomienda contar con una adecuada hidratación, tomar bebidas calientes con limón y miel, además de evitar el cigarrillo. El manejo farmacológico inicial se recomienda con dihidrocodeína (12.1 mg/5 mL): $5 \mathrm{~mL}$ cada 8 horas o codeína (30 mg cada 8 horas). Si el síntoma es severo se puede usar una infusión de morfina igual que para el manejo de disnea.

\section{Dolor}

Este puede presentarse debido a las comorbilidades existentes o como resultado de la tos excesiva e inmovilidad. Se debe evaluar con escala visual numérica de 0 a 10 .

Leve (1-3): AINEs: paracetamol en dosis habituales. Se debe reducir la dosis en ancianos frágiles, falla renal, elevación de transaminasas o peso $<50 \mathrm{~kg}$ (750 mg cada 8 horas). Moderado a severo (4-10): si el dolor persiste o empeora: asociar codeína $30 \mathrm{mg}$ cada 6 a 8 horas por horario. Dosis máxima: $120 \mathrm{mg} /$ día. Si se llega a la dosis máxima y el dolor persiste: suspender codeína. Considere iniciar opioide fuerte (morfina oral) a dosis de 3 a $4 \mathrm{mg}$ orales cada 4 a 6 horas. Si no tolera vía oral considerar inicio de morfina intravenosa. $S i$ el paciente consume diariamente opioides para el tratamiento de dolor crónico y tiene control de dolor y tolera la vía oral: continuar misma dosis. En caso de no tener control de dolor se recomienda el aumento de $20-25 \%$ de su opioide base y dejar rescates de $10 \%$ de la dosis total. En caso de pérdida de la vía oral rotación equianalgésica a opioide intravenoso.

\section{Fiebre}

Manejo farmacológico: se recomienda para el manejo sintomático el uso de acetaminofén oral o en caso de no tolerancia a la vía oral su uso intravenoso a dosis de $1 \mathrm{~g}$ cada 8 horas. Considerar reducción de dosis (ver dolor). En caso de refractariedad al síntoma puede considerarse el uso de metamizol (dipirona) a necesidad sin superar dosis de $5 \mathrm{~g}$ en 24 horas.

\section{Secreciones}

Las secreciones respiratorias pueden ser difíciles de controlar y alterar de manera importante la calidad de vida, particularmente en el contexto de patología pulmonar subyacente. 
Tabla 2: Pacientes atendidos por el Servicio de Cuidados Paliativos institucional durante la pandemia.

\begin{tabular}{|c|c|c|c|c|c|c|c|c|c|}
\hline Género & Edad & $\begin{array}{c}\text { Diagnóstico } \\
\text { patología de } \\
\text { base }\end{array}$ & $\begin{array}{l}\text { Diagnóstico } \\
\text { de COVID-19 }\end{array}$ & Espacio vital & ECOG & PPI & Síntomas & $\begin{array}{l}\text { Tratamiento- } \\
\text { multidisciplinario }\end{array}$ & Desenlace \\
\hline Femenino & 97 & EPOC & + & Frágil & 2 & Mayor de 6 & Disnea & Morfina infusión & $\begin{array}{l}\text { Muerte sin } \\
\text { sufrimiento }\end{array}$ \\
\hline Femenino & 90 & $\begin{array}{l}\text { Neumonía } \\
\text { Alzheimer }\end{array}$ & + & Dependiente & 4 & Mayor de 6 & $\begin{array}{l}\text { Disnea } \\
\text { refractaria }\end{array}$ & Sedación paliativa & $\begin{array}{l}\text { Muerte sin } \\
\text { sufrimiento }\end{array}$ \\
\hline Masculino & 79 & Poliartrosis & - & Robusto & 2 & Mayor de 6 & Dolor & Morfina oral & Domicilio \\
\hline Masculino & 50 & Melanoma IV & + & $\begin{array}{l}\text { Menor de } 65 \text { años } \\
\text { con patología } \\
\text { crónica }\end{array}$ & 1 & Mayor de 6 & Disnea & Morfina infusión & $\begin{array}{l}\text { Muerte sin } \\
\text { sufrimiento }\end{array}$ \\
\hline Femenino & 90 & Neumonía & + & Frágil & 4 & Menor de 6 & Disnea & Morfina infusión & $\begin{array}{l}\text { Muerte sin } \\
\text { sufrimiento }\end{array}$ \\
\hline Masculino & 47 & Pancreatitis & - & $\begin{array}{l}\text { Menor de } 65 \text { años } \\
\text { con patología } \\
\text { crónica }\end{array}$ & 0 & Menor de 6 & Tos y dolor & Acetaminofén-codeína & Domicilio \\
\hline Masculino & 67 & Cáncer de próstata & - & Frágil & 4 & Mayor de 6 & Disnea & Morfina infusión & $\begin{array}{l}\text { Muerte sin } \\
\text { sufrimiento }\end{array}$ \\
\hline
\end{tabular}

ECOG = Eastern Cooperative Oncology Group. PPI = Palliative Prognostic Index (Índice de pronóstico paliativo).

Fuente: los autores.

Bromuro de hioscina: ampollas $20 \mathrm{mg} / \mathrm{mL}$, tabletas de $10 \mathrm{mg}$, dosis: 10-20 mg IV cada 8 horas, 10 mg VO cada 8 horas.

\section{Delirium $^{(8)}$}

Siempre usar estrategias de prevención no farmacológicas como: recordar fecha, hora, lugar, hacer uso de claves externas como calendarios o relojes, mantener cerca dispositivos de ayuda visual y/o auditiva, evitar restringir el movimiento a menos que sea absolutamente necesario, orientación regular, evitar el estreñimiento, mantener la oxigenación y evitar la retención urinaria.

En caso de delirium hiperactivo se recomienda haloperidol a dosis de 0.5-1 mg IV/SC a titular y luego uso por horario cada 8 horas si los síntomas son persistentes (dosis máxima $15 \mathrm{mg}$ / día), tener en cuenta que éste es un medicamento que prolonga el intervalo QT. En el contexto de dificultad respiratoria severa y al final de la vida se debe usar un sedante ansiolítico como midazolam para permitir una titulación rápida, si es necesario usar midazolam 2.5-5 mg IV/SC titular y luego uso por horario en caso de ser persistente ${ }^{(9)}$. En caso de síntomas refractarios (delirium, disnea, dolor) seguir indicaciones de sedación paliativa (Tabla 2).

\section{Sedación paliativa}

Se entiende por sedación paliativa «la administración deliberada de fármacos, en las dosis y combinaciones requeridas, para reducir la conciencia de un paciente con enfermedad avanzada o terminal, tanto como sea preciso para aliviar adecuadamente uno o más síntomas refractarios y con su consentimiento explícito, implícito o delegado» ${ }^{(10)}$. En pacientes con COVID-19 que tengan mal control de síntomas y no son candidatos a ingreso en unidades de cuidado intensivo o están bajo ventilación mecánica invasiva en contexto de fracaso terapéutico, la sedación paliativa se convierte en una alternativa para el manejo de síntomas refractarios ${ }^{(11)}$.

\section{PROTOCOLO SEDACIÓN PALIATIVA}

- Discutir caso dentro del equipo tratante y confirmar que existe acuerdo respecto del uso de sedación paliativa.

- Mantener fármacos para el control de síntomas (analgésicos, antipsicóticos y otros).

- Se recomienda la suspensión de hidratación y/o alimentación artificial, no poner sondas nasogástricas.

- Suspender intervenciones que no aporten al confort y control de síntomas (monitorización, profilaxis farmacológicas y otros).

- Monitorizar frecuentemente que se mantenga el nivel de sedación requerido ${ }^{(12,13)}$.

- Se recomienda como primera elección el uso de midazolam. Iniciar con un bolo de 3-5 mg de midazolam, vía subcutánea o intravenosa lenta, y continuar en infusión continua $2 \mathrm{mg} / \mathrm{h}$. Si se precisa, por sedación insuficiente, se podrá subir el ritmo de infusión 0.5 o $1 \mathrm{mg}$ /hora hasta lograr control del síntoma. En cualquier momento se pueden administrar dosis de rescate subcutáneas o intravenosa lenta de $5 \mathrm{mg}$ en caso que persista sintomático ${ }^{(14-16)}$.

Intervenciones con la familia en la elaboración de duelo

Usamos la gestión basada en la comunicación, que incluye reconocer, responder y validar las respuestas emocionales en una 
estrategia clave para abordar el duelo anticipatorio entre pacientes críticos y sus familias; esta estrategia se realiza por todo el equipo multidisciplinario (psicología, psiquiatría, cuidados paliativos) ${ }^{(17)}$.

Tenemos en cuenta que el rito de la despedida es importante para la elaboración del duelo de la familia, pero por la contingencia no es posible ${ }^{(18)}$.

Generar un espacio al familiar o cuidador del paciente para permitirle llorar, para pensar en la persona que se ha marchado y lo que significaba para ellos, para expresar los sentimientos de culpa por no haber podido estar al lado del paciente y hacer énfasis en que las limitaciones las ha puesto la pandemia, ayudándolos a prepararse para la probable muerte es una parte crítica del trabajo de duelo anticipado, particularmente porque la falta de preparación es asociada con duelos patológicos ${ }^{(19)}$.

Es importante para la familia cercana y el cuidador principal sentir que no están solos y que están conectados, aunque sea de manera virtual por vid eoconferencias, espacios que se han generado en nuestra institución. Alentar a expresar la pena y el dolor con personas de confianza, amigos y familia. Sin embargo, el manejo de los síntomas y la calidad de la atención al final de la vida es generalmente mejor en orden: domicilio, hospicio en comparación con las muertes hospitalarias; la incidencia de ansiedad y la depresión es mayor al final de la vida en los pacientes hospitalizados ${ }^{(20)}$.

\section{Espiritualidad ante la presencia del COVID-19}

Tiene gran importancia el apoyo espiritual, pues ofrece protección contra la desesperación del final de la vida en aquellos para quienes la muerte es inminente ${ }^{(21)}$.

En nuestro caso para los pacientes con creencias religiosas católicas nuestro servicio institucional de capellanía brinda atención religiosa que ayuda a los pacientes que enfrentan enfermedades graves a sobrellevar mejor sus síntomas y su pronóstico. De acuerdo con las creencias religiosas, su intervención se centra en consolar al paciente y mejorar su bienestar en el contexto tanto del dolor espiritual como de la enfermedad crítica, deben explorarse con todos los pacientes y cuidadores principales. El servicio de cuidados paliativos debe abordar las necesidades de cuidado espiritual de aquellos pacientes que no están representados por la capellanía disponible o que no son religiosos ${ }^{(22)}$.

\section{CONCLUSIÓN}

Desde el 30 de marzo de 2020, iniciada la cuarentena en nuestro país y en nuestra capital, como lo muestra la Figura 1, en la institución se han atendido cerca de 110 pacientes con síntomas respiratorios sospechosos de COVID-19, de los cuales

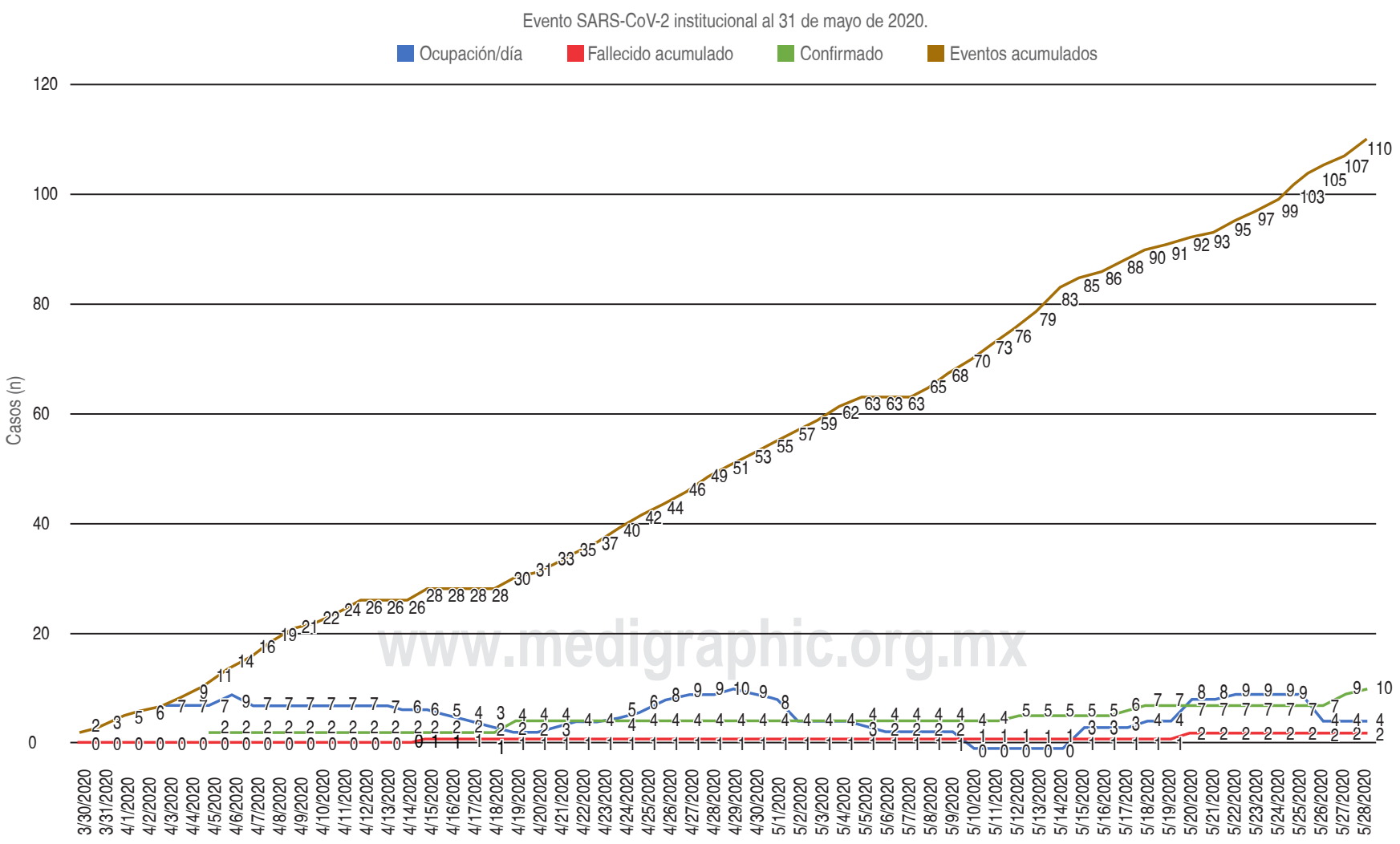

Figura 1: Gráfica de casos COVID-19 en la institución del 30-03-2020 al 30-05-2020. Fuente: los autores. 
fueron confirmados 16 pacientes, hasta el momento el Servicio de Cuidados Paliativos ha tratado de forma multidisciplinaria a siete pacientes, de los cuales cuatro fueron confirmados con la infección y dos de los verdaderos positivos murieron. El síntoma más importante que requirió paliación por nuestro servicio fue la disnea, la cual fue manejada exitosamente con morfina oral e intravenosa según el caso y uno de los pacientes requirió sedación paliativa (Figura 1).

Todos los pacientes y sus familias tuvieron soporte del servicio e intervención multidisciplinaria de psicología, psiquiatría, trabajo social y terapia ocupacional, al igual que acompañamiento religioso como parte del soporte espiritual, tratando adecuadamente síntomas y evitando sufrimiento. Los cuidados paliativos se convierten en una gran herramienta para brindar atención y apoyo a los pacientes, familiares y personal de la salud, controlando efectivamente síntomas, promoviendo la calidad de vida, ayudando en la toma de decisiones difíciles y brindando atención integral de la salud física, psicológica, social y espiritual.

\section{AGRADECIMIENTOS}

A la Asociación Colombiana de Cuidados Paliativos ACCP por su apoyo y soporte académico.

Responsabilidades éticas: Protección de personas y animales. Los autores declaran que para esta investigación no se han realizado experimentos en seres humanos ni en animales. Confidencialidad de los datos. Los autores declaran que han seguido los protocolos de su centro de trabajo sobre la publicación de datos de pacientes. Derecho a la privacidad y consentimiento informado. Los autores declaran que en este artículo no aparecen datos de identificación de pacientes.

Financiamiento para el estudio: Ninguno.

Fuente de financiamiento: Los autores declaran que no se recibió financiamiento de ninguna entidad gubernamental o privada.

Conflicto de intereses: Los autores declaran que no tienen conflicto de intereses que revelar.

\section{REFERENCIAS}

1. Trujillo-cariño AL, Allende-Pérez S, Verástegui-Avilés E. Utilidad del índice pronóstico paliativo (PPI) en pacientes con cáncer. Gac Mex Oncol. 2015;12:234-239.

2. Bajwah S, Wilcock A, Towers R, et al. Managing the supportive care needs of those affected by COVID-19. Eur Respir J. 2020;55:2000815.

3. Salako O, Okunade K, Allsop M, et al. Upheaval in cancer care during the COVID-19 outbreak. Ecancermedicalscience. 2020;14:ed97. doi: 10.3332/ecancer.2020.ed97.

4. Landman A, Feetham L, Stuckey D. Cancer patients in SARS-CoV-2 infection: a nationwide analysis in China. Lancet Oncol. 2020;2045:335-337.

5. Radbruch L, De Lima L, Knaul F, et al. Redefining palliative care-a new consensus-based definition. J Pain Symptom Manage. 2020. doi: 10.1016/j.jpainsymman.2020.04.027.

6. Fusi-Schmidhauser T, Preston NJ, Keller N, Gamondi C. Conservative management of COVID-19 patients-emergency palliative care in action. J Pain Symptom Manage. 2020;60:e27-e30. doi: 10.1016/j. jpainsymman.2020.03.030.

7. Sanders JM, Monogue ML, Jodlowski TZ, Cutrell JB. Pharmacologic treatments for coronavirus disease 2019 (COVID-19): a review. JAMA. 2020. doi: 10.1001/jama.2020.6019.

8. Mao L, Jin H, Wang M, et al. Neurologic manifestations of hospitalized patients with coronavirus disease 2019 in Wuhan, China. JAMA Neurol. 2020;77:1-9. doi: 10.1001/jamaneurol.2020.1127.

9. Roy-Byrne P, Sareen J, Mcintyre JS, Anzia DJ. Treatment of patients. Am Psychiatr [Internet]. 2010;1:1-90. Available in: http://www. psychiatryonline.com/pracGuide/pracGuideTopic_9.aspx.\%0AFor

10. Villavicencio-Chávez C, Sobrevia X, Esquirol-Caussa J, GuerreroAlgara L, Vaquero-Cruzado J, García-Navarro Y. Protocolo de actuación para la Sedación Paliativa ante una disnea refractaria en domicilio. Protocolo especial COVID-19. Barcelona. 27 de marzo de 2020. Disponible en: info@fundacioncaredoctors.org

11. Therefore $\mathrm{S}$. The importance of addressing advance care planning and decisions about do-not-resuscitate orders during novel coronavirus 2019 (COVID-19). JAMA. 2020;2019:2019-2020.

12. Borasio GD, Gamondi C, Obrist M, Jox R, For The Covid-Task Force Of Palliative Ch. COVID-19: decision making and palliative care. Swiss Med Wkly. 2020;150:w20233.
13. Hendin A, La Rivière CG, Williscroft DM, O’Connor E, Hughes J, Fischer LM. End-of-life care in the emergency department for the patient imminently dying of a highly transmissible acute respiratory infection (such as COVID-19). CJEM. 2020;1-4. doi: 10.1017/ cem.2020.352.

14. Lake MA. What we know so far: COVID-19 current clinical knowledge and research. Clin Med (Lond). 2020;20:124-127.

15. Kunz R, Minder M. COVID-19 pandemic: palliative care for elderly and frail patients at home and in residential and nursing homes. Swiss Med Wkly. 2020;150:w20235.

16. Shankar A, Saini D, Roy S, et al. Cancer care delivery challenges amidst coronavirus disease-19 (COVID-19) outbreak: specific precautions for cancer patients and cancer care providers to prevent spread. Asian Pac J Cancer Prev. 2020;21:569-573.

17. SECPAL. Recomendaciones para familiares para la despedida y el duelo ante la presencia del COVID-19. Recomendaciones para familiares para la despedida y el duelo ante la presencia del COVID-19. 2020; 8-9.

18. Etkind SN, Bone AE, Lovell N, et al. The role and response of palliative care and hospice services in epidemics and pandemics: a rapid review to inform practice during the COVID-19 pandemic. J Pain Symptom Manage. 2020;60:e31-e40. doi: 10.1016/j. jpainsymman.2020.03.029.

19. Wallace CL, Wladkowski SP, Gibson A, White P. Grief during the COVID-19 pandemic: considerations for palliative care providers. J Pain Symptom Manage. 2020;60:e70-e76. doi: 10.1016/j. jpainsymman.2020.04.012.

20. Wright AA, Keating NL, Balboni TA, Matulonis UA, Block SD, Prigerson HG. Place of death: correlations with quality of life of patients with cancer and predictors of bereaved caregivers' mental health. J Clin Oncol. 2010;28:4457-4464. doi: 10.1200/JCO.2009.26.3863.

21. Berning JN, Poor AD, Buckley SM, et al. A novel picture guide to improve spiritual care and reduce anxiety in mechanically ventilated adults in the Intensive Care Unit. Ann Am Thorac Soc. 2016;13:13331342.

22. Fausto J, Hirano L, Lam D et al. Creating a palliative care inpatient response plan for COVID-19-the UW Medicine Experience. J Pain Symptom Manage. 2020;60:e21-e26. doi: 10.1016/j.jpainsymman.2020.03.025. 


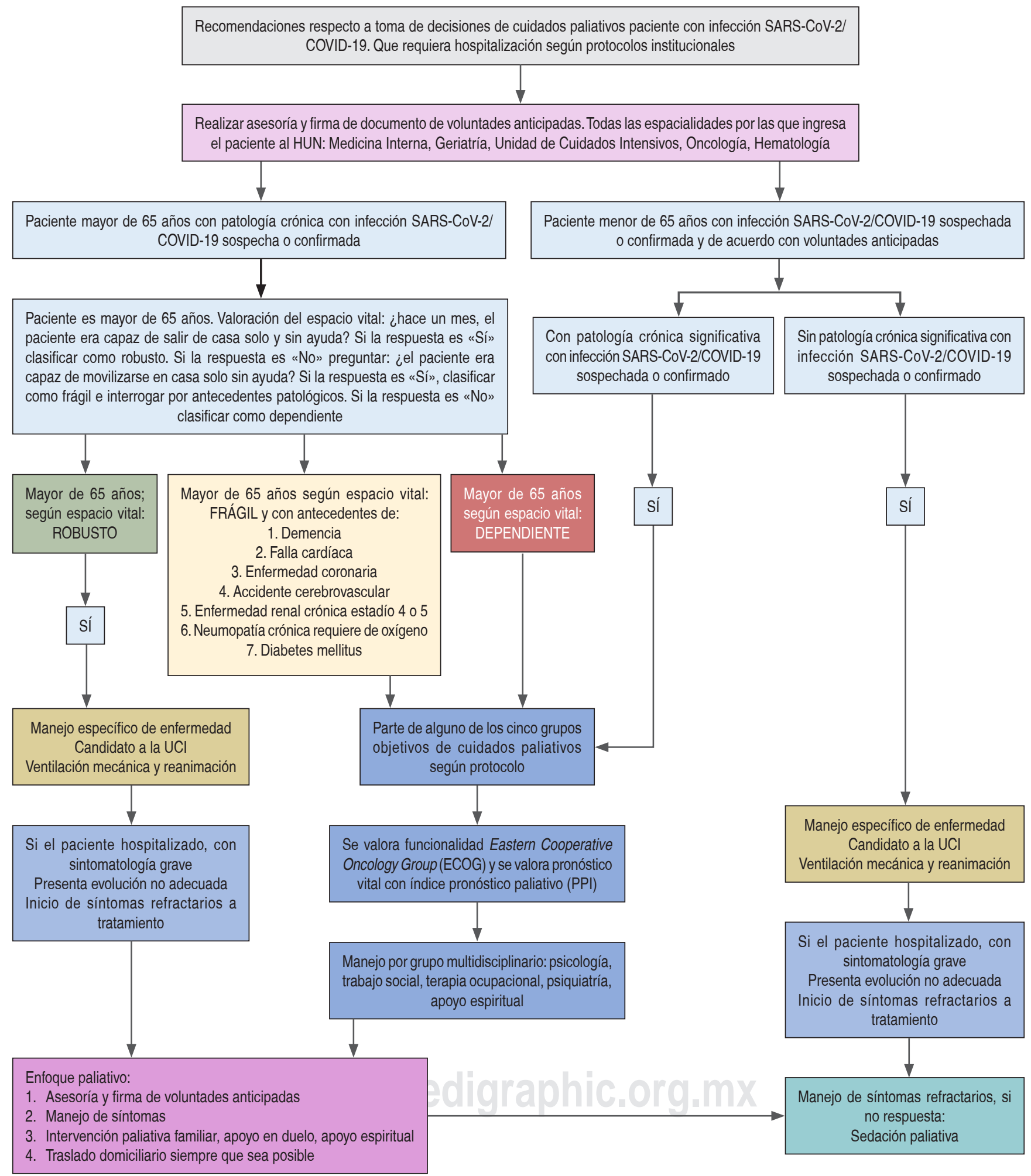

Anexo 1: Flujograma. Resumen del modelo de atención institucional. 